\title{
Sexual-Moral Attitudes of Chinese College Students: Two Moral Criteria Systems?
}

\author{
Yongkang Zhou ${ }^{1}$, Weixi Zeng ${ }^{2}$, Yalin $\mathrm{Hu}^{1}$ \\ ${ }^{1}$ School of Culture and Social Development Studies, Southwest University, Chongqing, China \\ ${ }^{2}$ School of Political Science and Public Administration, University of Electronic Science and Technology, \\ Chengdu, China \\ Email: zyk@swu.edu.cn, zwx213@hotmail.com, lindahuyalin@gmail.com
}

Received December 23 ${ }^{\text {rd }}$, 2013; revised January 25 $5^{\text {th }}$, 2014; accepted February $18^{\text {th }}, 2014$

\begin{abstract}
Copyright (C) 2014 Yongkang Zhou et al. This is an open access article distributed under the Creative Commons Attribution License, which permits unrestricted use, distribution, and reproduction in any medium, provided the original work is properly cited. In accordance of the Creative Commons Attribution License all Copyrights (c) 2014 are reserved for SCIRP and the owner of the intellectual property Yongkang Zhou et al. All Copyright (C) 2014 are guarded by law and by SCIRP as a guardian.
\end{abstract}

\begin{abstract}
The aim of this study is to develop a valid scale to measure the construct of the sexual-moral attitudes of Chinese college students. Based on open questionnaire investigation, in-depth interviews, exploratory factor analysis and confirmatory factor analysis, the 20-item Sexual-Moral Attitudes Scale (SMAS) was developed and emerged with good psychometric properties. The multi-hierarchy and multi-dimension construct was confirmed. It can be concluded that contemporary Chinese college students has two moral criteria systems to regulate their sexual behaviors. The first one is the Basic Sexual-Moral Principles, which includes two first-hierarchy dimensions as Sense of Responsibility and Sense of Ethics; the second one is Strict Sexual-Moral Principles, which includes three first-hierarchy dimensions as Being in Love, Chastity and Fidelity.
\end{abstract}

Keywords: Sexual-Moral Attitudes; Two-Hierarchy Construct; Chinese College Students; Validity

\section{Introduction}

Sexual-moral attitudes regulate people's sexual behaviors and have relevance to individuals' love style and mate selection (Lacey \& Reifman, 2004). Sexual-moral attitudes are different among diverse cultural environments (Villarreal, 1998; Harold \& Edward, 2002; Beckwith \& Morrow, 2005) and change with time (Kinsman, 1997; Hamburger \& Merle, 1996). Since the Open Door policies in the 1970s and the economic reforms of the 1980s, attitudes towards sexuality in China have changed (Zhang \& Beck, 1999), premarital sexual intercourse appears more and more frequently among young people and people in China are now more tolerant toward extramarital sexual behaviors. In some former studies, the sexual attitudes in the Chinese have been described by scholars as suppressive or nonsuppressive, based on observations made on various aspects of the Chinese culture (Ng \& Lau, 1990). During the age of information and globalization, sexual information, such as porn photographs and audio-visual products, is easy to get on mass media, and some western sexual attitudes are widely known by Chinese, especially Chinese college students. So the traditional sexual-moral attitudes of Chinese people would have been changed by the new shocks and it is necessary to exam the construct of sexual-moral attitudes of contemporary Chinese people, especially Chinese college students, the most sensitive and fashionable group in China. We premise that traditional and some modern or western values both have effects on the sexualmoral attitudes of Chinese college students; and they may select some beneficial sexual-moral attitudes to decrease the inner conflicts when they have experienced some controversial sexual behaviors. The aim of this study is to develop valid scale to measure the complicated constructs of the sexual-moral attitudes of Chinese college students.

\section{Methods}

\section{Procedure}

Based on open questionnaire investigation, in-depth interviews and content analysis, the initial 31 items of Sexual-Moral Attitudes Inventory of Chinese College Students (SMAI) were developed. It is similar to The Brief Sexual Attitudes Scale (Hendricks \& Reich, 2006). Participants responded to the SMAI items using a 5-point, Likert-type scale that ranged from $1=$ strongly disagree to 5 = strongly agree, each item is the answer to the question: if we want to have sexual intercourse with others, what principles should we follow?

Using exploratory factor analysis, the first-hierarchy factors included five dimensions were found; after the first exploratory factor analysis step, about 11 items were deleted according to some statistical principles. The formal inventory includes 20 items. Then using exploratory factor analysis to the five firsthierarchy factors, two higher-hierarchy factors were found. Using confirmatory factor analysis, the two-hierarchy SexualMoral Attitudes model was tested.

\section{Measures}

Questionnaires included measures of the sexual-moral atti- 
tudes (with 20 items), and demographic information on participants' gender, sexual orientation (heterosexual or homosexual), age, whether experienced sexual intercourse and when they experienced the first sexual intercourse and The Brief Sexual Attitudes Scale (Hendrick, 2006) was employed to test the validity.

\section{Participants}

A total of 1102 unpaid undergraduate students from five Chinese universities participated in the survey (either as volunteer or as part of a course requirement); all responses were anonymous. This sample was divided into two parts, one for exploratory factor analysis (361 males and 280 females, had an age range of 16 - 26 years, $M=20.12, S D=1.53$ ), and the other for confirmatory factor analysis (290 males and 171 females, had an age range of $16-27$ years, $\mathrm{M}=21.38$, SD = 1.81). In this sample, 462 (42\%) had experienced sexual intercourse. 71 (6.5\%) reported homosexual orientation.

\section{Results}

\section{Exploratory Factor Analysis}

Principal components analysis using varimax rotation was conducted on the Sexual-Moral Attitudes Scale (SMAS). Five components having eigenvalues greater than 1.0 were extracted by PCA (explaining $45.82 \%$ of variance). The factor loadings are showed in Table 1. Loadings of less than .40 are suppressed. The first factor (f1) has 5 items, they represent some basic principles that must be complied with when sexuality happens, so f1 can be generalized as Sense of Responsibility; as the same, f2 (4 items) can be named Being in Love; f3 (4 items) reflects the principle of Sense of Ethics, f4 (3 items) can be generalized as Chastity and f5 (4 items) can be named as Fidelity.

In order to find higher hierarchy factors, principal components analysis using varimax rotation was conducted on the five first-hierarchy factors. Two components having eigenvalues greater than 1.0 were extracted by PCA (explaining $54.27 \%$ of variance). The factor loadings are showed in Table 2 . Loadings of less than .40 are suppressed. The first higher-hierarchy factor (F1) includes the first-hierarchy factors as follows: Sense of Responsibility ( $f 1)$ and Sense of Ethics ( $f 3)$, they both reflect some basic principles that must be complied with when sexuality happens, so F1 can be generalized as Basic Sexual-Moral Principles; The second higher-hierarchy factors (F2) includes Being in Love (f2), Chastity (f4) and Fidelity (f5), they reflect some higher moral criteria, so F2 can be generalized as Strict Sexual-Moral Principles.

\section{Reliability}

For the Sexual-Moral Attitudes Scale, the Cronbach’s alpha

Table 1.

Loadings of 20 items of SMAI in five first-hierarchy factors.

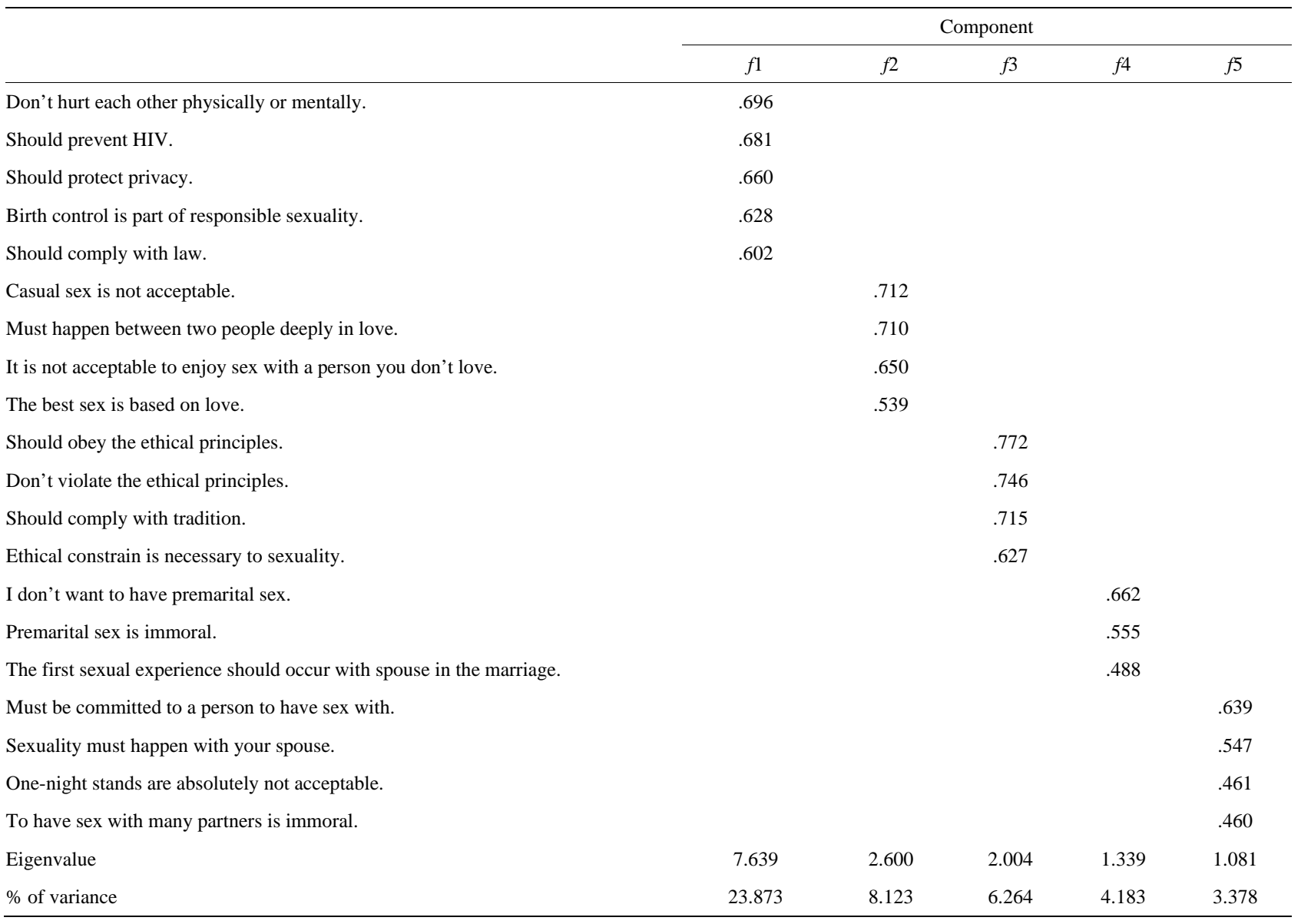


Table 2.

Loadings of five first-hierarchy factors in two second-hierarchy factors.

\begin{tabular}{lll}
\hline & F1 & F2 \\
\hline Sense of responsibility $(f 1)$ & .819 & \\
Sense of ethics $(f 3)$ & .797 & \\
Being in love $(f 2)$ & & .811 \\
Fidelity (f5) & & .713 \\
Chastity (f4) & & .604 \\
Eigenvalue & 2.919 & 1.423 \\
\% of variance & 36.490 & 17.789 \\
\hline
\end{tabular}

values were as follows: Sense of Responsibility $=0.81$; Being in Love $=0.76$; Sense of Ethics $=0.73$; Chastity $=0.68$; Fidelity $=0.78$. Internal consistency for the scale was 0.79 . Spearman's correlation of test and retest data, indicated high levels of consistency over time, with a significant relationship for the overall scores obtained for two tests $(r=.823, p<.01)$. Ten correlations between the five factors were modest (ranging from 0.23 to 0.54 ).

\section{Concurrent Validity}

Strict Sexual-Moral Principles (F2) had strong negative correlation with Permissiveness $(r=-.81, p<.05)$ and Instrumentality $(r=-.76, p<.05)$ dimensions, and correlated positively with Birth Control $(r=.61, p<.05)$ and Communion $(r$ $=.84, p<.05)$ dimension in The Brief Sexual Attitudes Scale (Hendrick, 2006). Basic Sexual-Moral Principles (F1), score of which distributed with obvious negative skewness, had significant positive correlation with Birth Control $(r=.72, p<.05)$ and didn't have significant correlation with the other three dimensions.

\section{Construct Validity}

Confirmatory factor analyses were conducted to test the construct validity. The one-hierarchy model with five factors had a GFI of .95, AGFI of .92, CFI of .88, NFI of .87, RMSEA of .094 , and $\chi^{2} / d f=12.85$, and the two-hierarchy model with five first-hierarchy factors and two second-hierarchy factors had a GFI of .98, AGFI of .95, CFI of .96, NFI of .94, RMSEA of .061 and $\chi^{2} / d f=3.05$. So the two-hierarchy model had better psychometric properties than the one-hierarchy model.

\section{Difference Analysis}

The mean score for the 20 items in the SMAS was 66.24 (SD = 6.51). There was no difference between male and female responses, but there was difference between college students who have experienced sexual intercourse and those who have not experienced sexual intercourse $(\mathrm{t}=-5.16, p<0.01)$. There was no difference between male and female responses to F1, but There was difference between male and female responses to F2 $(\mathrm{t}=-2.31, p<0.05)$. College students who have experienced premarital sexual intercourse and those who have not experienced premarital sexual intercourse response to F2 differently $(\mathrm{t}=-7.42, p<0.01)$ but not differently to F1. There was no significant difference in every dimension's mean score of SMAS between The homosexual sample and the heterosexual sample.

\section{Discussions}

In the present study, the 20-item Sexual-Moral Attitudes Scale was developed to measure the moral aspects of sexual attitudes of Chinese college students. Based on EFA and CFA, a multi-hierarchy and multi-dimension model was confirmed. Results from reliability and validity analysis indicated that the scale emerged with good psychometric properties. It can be concluded that contemporary Chinese college students has two moral criteria systems to assess their sexual behaviors. The first one is the Basic Sexual-Moral Principles, which includes two first-hierarchy factors as Sense of Responsibility and Sense of Ethics, no matter having experienced premarital sexual intercourse or not, Chinese college students have strong agreement on this moral criterion. The second one is Strict Sexual-Moral Principles, which includes three first-hierarchy factors as Being in Love, Chastity and Fidelity; they are strict moral criteria and would be complied with just by those students who haven't experienced premarital sexual intercourse. In the case study, some participants reported their anti-tradition sexual behaviors, such as one-night stands and have sexual relationships with several partners; some of them also wanted to protect the Strict Sexual-Moral Principles, but seriously affected by the easygetting sexual information, their sexuality was out of control; these participants inclined to emphasize the Instrumentality (Hendrick, 2006) and choose to agree with the Basic SexualMoral Principles as coping pattern to reduce the inner conflict. This research has some limitations. First, participants were university students; thus, the generalizability may be limited and the scale's utility to other age groups should be in caution. In addition, the future study should provide more information about the correlation between sexual-moral attitudes and mental health. Now, the two moral criteria systems have been shown in the Chinese culture.

\section{REFERENCES}

Beckwith, H. D., \& Morrow, J. A. (2005). Sexual attitudes of college students: The impact of religiosity and spirituality. College Student Journal, 39, 357-367.

Hamburger, M. E. (1996). Sexual attitudes through the ages. The Journal of Sex Research, 33, 162-164.

Harold, E. S., \& Edward, S. (2002). Sexuality in America: Understanding our sexual values and behavior. Archives of Sexual Behavior, 31, 383-385. http://dx.doi.org/10.1023/A:1016240829935

Hendricks, C., Hendricks, S., \& Reich, D. A. (2006). The brief sexual attitudes scale. The Journal of Sex Research, 43, 76-87. http://dx.doi.org/10.1080/00224490609552301

Kinsman, G. (1997). Invented moralities: Sexual values in an age of uncertainty. The Canadian Review of Sociology and Anthropology, 34, 478-481.

Lacey, R. S., Reifman, A., \& Scott, J. P. (2004). Sexual-moral attitudes, love styles, and mate selection. The Journal of Sex Research, 41, 121-124. http://dx.doi.org/10.1080/00224490409552220

Ng, M. L., \& Lau, M. P. (1990). Sexual attitudes in the Chinese. Archives of Sexual Behavior, 19, 373-388. http://dx.doi.org/10.1007/BF01541932

Villarreal, A. M. (1998). Cultural influences on the sexual attitudes, beliefs, and norms of young Latin adolescents. Journal of the Society of Pediatric Nurses, 3, 69-80. http://dx.doi.org/10.1111/j.1744-6155.1998.tb00030.x

Zhang, K., Li, D., \& Beck, E. J. (1999). Changing sexual attitudes and behavior in China: Implications for the spread of HIV and other sexually transmitted diseases. Aids Care, 11, 581-589. http://dx.doi.org/10.1080/09540129947730 